\title{
Clinical Training in Music Therapy :
}

\section{Perceptions of Preparedness and Satisfaction}

\author{
Edward A. Roth ${ }^{12}{ }^{*}$, Xueyan Hua ${ }^{1}$, Wang Lu ${ }^{1}$, Jordan Blitz Novak ${ }^{1}$, Fei Wang ${ }^{1}$, \\ Taylorlyn N. Mehnert ${ }^{12}$, Rebekah K. Morano ${ }^{12}$, Jennifer Fiore ${ }^{1}$, \\ Alycia J. Sterenberg Mahon ${ }^{12}$ \\ 1 Department of Music Therapy, School of Music, Western Michigan University \\ 2 Brain Research and Interdisciplinary Neurosciences Lab \\ *edward.roth@wmich.edu
}

Received: 8 June 2020 Accepted: 14 October 2021 Published: 1 November 2021

Editor: Susan J Hadley Reviewers: ChihChen Lee, Debbie Bates

\begin{abstract}
Objective: This paper examines the experiences of music therapy students throughout their clinical training. Three surveys inquired about: 1) the perception from both interns and supervisors as to interns' needs, 2) interns' preparedness, their skills, their priorities when choosing an internship, and whether their expectations for training were met (with comparisons between American and International respondents), and 3) satisfaction with clinical training. Method: Three separate surveys were distributed. The first survey's respondents included pre-interns $(n=19)$ and internship supervisors $(n=14)$ who had completed their training in the Great Lakes Region of the United States. The second survey's respondents included American interns ( $n=$ $50)$, American professionals $(n=353)$, International interns $(n=12)$, and International professionals $(n=50)$. Respondents for the third survey included professional music therapists who completed their curriculum in the United States and held the MT-BC professional credential $(\mathrm{N}=777)$. Results: Some differences between interns' and supervisors' perceptions of the interns' needs were found in Survey 1; significant differences were found between the preparedness and strengths/weaknesses between groups in Survey 2; and Survey 3 found general satisfaction with training with some areas respondents felt needed improvement. Conclusions: While there is overall satisfaction with training for music therapists, there are inconsistencies in students' experiences in, and perceptions of, their training.
\end{abstract}

Keywords: music therapy, clinical training, needs, preparedness, expectations, satisfaction 


\section{Literature Review}

\section{Music Therapy Curriculum}

Beginning in 1919, music therapy training in the United States was offered informally by pioneers such as Margaret Anderton, Isa Maude Ilsen, Harriet Seymour, Eva Vescelius, (Davis \& Gfeller, 2008) and Wilhelmina Harbert (Davis et al., 2018). Each had their own curriculum, philosophy for conducting music therapy, and indications of what instruments and/or types of music were most or least appropriate (de l'Etoile, 2000). Students were trained to work in hospitals and schools to address physical and mental needs of those with whom they worked (Davis et al., 2018). The first structured music therapy course was taught by Anderton and later by Ilsen at Columbia University starting in 1919 (de l'Etoile, 2000). The first recognized undergraduate and graduate university training programs were established at Michigan State University in 1944 and the University of Kansas in 1946, respectively (de l'Etoile, 2000). Due to the variety of training occurring across the country, the need arose to establish education standards to further the recognition of the profession.

With assistance from Music Teachers National Association, the National Association for Music Therapy (NAMT) was established in 1950 with the purpose to progress music therapy education and training, and determine standards for music therapy practice (Davis et al., 2018). This effort culminated in the undergraduate curriculum being formalized in 1952 (de l'Etoile, 2000), based on specific required music therapy courses and nationally rostered internships occurring outside the university setting (de l'Etoile, 2000). NAMT credentialed music therapists from their programs as Registered Music Therapists (RMT), with NAMT maintaining a roster of credentialed professionals within the association. Over time, philosophical differences regarding education and training arose within the music therapy community, which led to a split in the profession and the formation of the Urban Federation for Music Therapists (later renamed the American Association of Music Therapy [AAMT]) in 1971 (Davis et al., 2018). AAMT addressed education and training more flexibly through the use of professional competencies instead of required courses, and university-affiliated internships that were more closely related to the university (Davis et al., 2018). Music therapists who studied at AAMT programs were credentialed as Certified Music Therapists (CMT) or Advanced Certified Music Therapists (ACMT), with AAMT maintaining a roster of credentialed professionals for that association. In order to have one set of standards for credentialing music therapists, the Certification Board for Music Therapists (CBMT) was established in 1983 to serve as the single credentialing agency for the profession (CBMT, n.d.), consolidating credentials issued by NAMT and AAMT (AMTA, 2011). CBMT administered the first board-certification exam in 1985, which granted candidates the credential of board-certified music therapist (MT-BC). The MT-BC credential verifies that candidates meet the required knowledge to practice music therapy, and requires the professional to engage in 100 hours of continuing education over five years to maintain MT-BC status.

\section{Background on AMTA Professional Competencies}

The presence of two professional associations (NAMT and AAMT) resulted in confusion for the public and the profession, and limited the growth of the profession (Davis et al., 2018). Members from both associations voted to unify as one association in 1996, with ratification occurring in 1998, which formed the American Music Therapy Association (AMTA). AMTA established the Commission on Education and Training to study and design education and training standards for the new association. The use of AAMT's competency-based education was adopted (AMTA Professional Competencies, Adopted 1999, Revised 2013), with both nationally rostered and university-affiliated internships being offered (AMTA, 2011). The credentialed registries from both NAMT and AAMT were maintained through 2020 for the RMT, ACMT, and CMT credentials; while the MT-BC became established as the sole credential for the profession (AMTA, 
2011). The approved AMTA competency-based curriculum was designed to impart entry-level competencies in music foundations (45\%), clinical foundations (15\%), music therapy foundations and principles (15\%), general education (20-25\%), and other electives (5\%) (AMTA Standards for Education and Clinical Training, Adopted 2000, Revised 2017), while allowing each college/university program diversity in determining how to address competencies.

A bachelor's degree in music therapy addresses three main areas: 1) music foundations, 2) clinical foundations, and 3) music therapy foundations and principles. Each main category is comprised of several subcategories to be addressed through coursework, pre-internship clinical hours, and internship (AMTA Professional Competencies, Adopted 1999, Revised 2013). Since academic programs across the country vary in training styles, course requirements, and pre-internship clinical experiences, students may enter internship with different skill sets than peers from other institutions.

\section{Internship}

In addition to coursework, music therapy students are required to complete a minimum of 1,200 hours of clinical training through fieldwork and an internship. This involves music therapy students completing 180 hours in pre-internship experiences (practicum assignments from academic programs) and at least 900 hours of internship experience (AMTA Standards for Education and Clinical Training, Adopted 2000, Revised 2017). All AMTA-approved colleges and universities follow the above standard; however, depending on their philosophical orientation, coursework requirements for students may vary between institutions. The AMTA Professional Competencies are addressed through both academic courses and in the development of a training plan and written internship agreement. The academic training program periodically assesses students' progress through coursework and clinical training, and collaborates with the internship site to determine when a student has met all of the competencies to graduate (AMTA, Standards for Education and Clinical Training, Adopted 2000, Revised 2017). Upon completion of AMTA-approved academic training and internship, students are eligible to sit for the board certification exam administered by CBMT.

\section{Differences Between American \& International Students}

AMTA has a total of 88 accredited educational programs, which provides many opportunities for international students to study music therapy in the United States (AMTA Organization Directory Search, 2019). For international students, internship can be a difficult part of their academic experience. International students have additional challenges when studying outside of their home country due to unfamiliar learning contexts, differing learning styles, language barriers, and cultural differences, among other issues (Ruhanen et al., 2013). These differences can produce acculturative stress in international students not experienced by Americans, which can potentially have a negative impact on clients if such stress affects the well-being of interns during their internship experiences (Kim, 2011). The present research further explores these differences between American and international students.

\section{Internship Director Perceptions of Incoming Interns on AMTA Professional Competencies}

At the start of the internship, the supervisor may notice some significant deficiencies in the intern's ability to perform their job. If a pre-intern shows signs of severe professional competency problems prior to the start of the internship, the individual can be identified by academic faculty. However, sometimes these students move on to internships, nonetheless. In a survey of 32 academic program directors and 77 internship directors, "inadequate music skill development" was rated by both groups as the top indicator of students with severe professional competency problems. The same survey identified additional deficiencies that included poor musical skills, ethical violations, 
poor boundaries, and social inappropriateness (Hsiao, 2014). In a survey of 96 internship directors, respondents rated the majority of their interns as below average competency level for guitar, keyboard, percussion, and improvisational skills at the start of their internship (Jenkins, 2013). In another survey, 94 internship directors were asked to identify the most difficult aspects of supervision; "making sure competencies were met" was rated as one of the two top-ranked responses (Tanguay, 2008). The present research explores the different perceptions of internship directors and incoming interns on AMTA Professional Competencies.

\section{Professional Preparedness}

Since the music therapy curriculum was revised by NAMT in 1996, there have been few studies focused on educational satisfaction related to music therapy. One study in occupational therapy surveyed current students and graduates about professional education and preparedness (Hodgetts et al., 2007). The study indicated that students and recent graduates from occupational therapy felt that they lacked technical and intervention skills, yet both students and graduates were satisfied with their education.

\section{Research Questions}

An understanding of all of these factors - interns' preparedness, their skill in relation to the AMTA Professional Competencies, their satisfaction with their training, and the perception of both the intern and the supervisor as to the intern's needs - is necessary to give a full picture of the system used to train music therapists in the United States. In addition, understanding a student's growth from internship into the professional world for both American and International interns can help to improve the quality of training and provide support where needed. The questions below served as the primary research questions across the surveys administered:

Survey 1:

- What are the similarities and differences in how pre-interns and internship supervisors perceive areas of need within the AMTA Professional Competencies in terms of a) music foundations and b) clinical foundations?

\section{Survey 2:}

- What are the differences between American and International interns and professionals in clinical training in terms of a) AMTA Professional Competencies, b) general strengths and weaknesses, c) choosing an internship, and d) strengths and weaknesses of their internship?

\section{Survey 3:}

- How satisfied are music therapists with their undergraduate or equivalency curriculum as preparation for professional practice?

\section{Methods}

\section{Survey 1}

The purpose of this survey was to investigate the differences in how pre-interns and internship supervisors perceive areas of need within the AMTA Professional Competencies. The study was approved by an institutional review board ${ }^{i}$. The pre-interns were required to have completed at least two semesters of music therapy practicum courses to participate, while internship supervisors were required to be board-certified music therapists who had supervised at least one intern in the past two years. A list of internship supervisors was compiled using the AMTA National Roster website. Using purposive sampling, pre-interns were contacted through the Great Lakes Region (GLR) Music Therapy Association, GLR Student Association, and university faculty. The survey was made available in September 2016 for a duration of a month. The survey was a closed-form questionnaire developed by Knight (2008) with 36 Likert-scale rating 
(5-level) questions. The participants rated their need for assistance from "Little or no need for assistance" [1] to "Very high need for assistance in this area" [5]. Questions included inquiries about clinical foundations, music therapy, and music foundations competencies. In order to clarify the language for the participants, the phrase "concern for assistance" was changed to "need for assistance," "diagnosing client needs" was changed to "assessing client needs," and "closing a knowledge gap between academic program and internship" was added to the end of the first section of the questionnaire. The survey asked pre-intern respondents to rate their perceived need for assistance and asked internship supervisors to rate their own perception of the needs of their typical intern to meet the AMTA Professional Competencies. Between-group responses were compared using t-tests and Mann-Whitney tests.

\section{Survey 2}

The purpose of this study was to investigate internship preparedness and the differences between International and American music therapy interns and professionals. The study was approved by an institutional review board. The inclusion criteria required interns to have completed the first half of their internship and professionals to have earned their MT-BC credential within the previous five years (no earlier than March 2012). The investigators requested email addresses from the Certification Board for Music Therapists for professional music therapists and accessed the approved internship sites list posted on the AMTA website to obtain contact information for clinical training directors. The survey was open for one month. Participants who completed the survey were entered into a drawing for \$10 Amazon gift cards.

The survey instrument was created for this study by the investigators. It contained 13 questions, formatted as yes or no, multiple-choice, Likert-scale rating (4-level), and short answer. Five of these questions were modified from surveys used in earlier studies (Knight, 2008; Madsen \& Kaiser, 1999; Young, 2009). The survey included questions on demographics, how respondents decided where to complete their internship, perceived strengths and weaknesses in accordance with the AMTA Professional Competencies, and how those competencies were met during their internship. Response items were based on the major categories of the AMTA Professional Competencies and were used by permission from the Western Michigan University (WMU) music therapy pre-internship self-evaluation. Chi-square analyses were used to compare the four different groups with different sample sizes.

\section{Survey 3}

The purpose of this study was to examine the satisfaction level of undergraduate and equivalency students who have completed their degree program, with respect to their professional education and preparedness for practice in the United States. The study was approved by an institutional review board. The inclusion criteria for participants in this study required that participants be professional music therapists who completed their undergraduate degree or an equivalency curriculum in the United States and held the professional credential of MT-BC at the time of data collection. Participant emails were obtained from the CBMT email list for music therapists who held an MTBC credential, and the survey was open from October 16th, 2017 - November 16th, 2017. The survey contained one main research question with 20 sub-survey questions. Questions were modified from Hodgetts et al. (2007) and included yes or no, multiple choice, and Likert-scale rating questions (6-levels which are totally satisfied [6], mostly satisfied [5], somewhat satisfied [4], somewhat dissatisfied [3], mostly dissatisfied [2]; and totally dissatisfied [1]). Chi-square and Spearman correlation analyses were used to answer primary research questions. 


\section{Results}

\section{Survey 1}

Approximately 70 individuals were contacted for this study and 33 self-selected to enroll, including pre-interns $(n=19)$ and internship supervisors $(n=14)$ in the Great Lakes Region (GLR) of AMTA. Pre-interns had an age range of 18-25 with two males (10.53\%), 16 females (84.21\%), and one who identified with the "other" category (5.26\%). Internship supervisors in this study had an age range of 25-55 with one male (7.14\%) and 13 females (92.86\%). Determining the varying levels of perceived need required to meet the AMTA Professional Competencies by both students and internship supervisors is an important step to improving the quality of internship training. In this survey, the means of the music therapy pre-interns and internship supervisors were compared across 36 questions regarding clinical foundations and music foundations using a t-test and Mann-Whitney test (Field, 2013) utilizing the program SPSS.

\section{Level of Need for Assistance in Clinical Foundations}

The clinical foundations portion of the AMTA Professional Competencies covers skills used to facilitate a therapeutic session and knowledge required to be a successful clinician. Identifying the level of need for incoming interns in this area is a valuable tool for the internship supervisors to use when designing their internship program. The mean scores of pre-interns and internship supervisors were calculated on each item to find areas requiring the highest level of need. The surveyed pre-interns were moderately to highly concerned about "finding out what is expected of me as an intern" $(M=3.526)$, "closing the knowledge gap between academic program and internship" $(M=3.368)$, "understanding the facility's intern evaluation process" $(M=3.526)$, and "obtaining instructional resources and materials" ( $M=3.053)$. In contrast, the internship supervisors were moderately to highly concerned about "facilitating group discussions" ( $M$ = 3.714), "assisting students in assessing client needs" $(M=3.571)$, "dealing with stress" $(M=3.5)$, and "closing the knowledge gap between academic program and internship" ( $M=3.357)$. "Closing the knowledge gap between academic program and internship" was the only commonality between the pre-interns and the internship supervisors. The mean scores of the internship supervisors were compared against the mean scores of the pre-interns using the independent t-test. The independent t-test was used to compare the mean scores due to the assumed normality of data distribution, independence and homogeneity. One area was found to be statistically significant: "understanding the facility's intern evaluation process," $t(31)=4.217, p=.001$ with an effect size of 1.514. See Table 1 for remaining statements.

\section{Level of Need for Assistance in Music Foundations}

The music foundations section of the AMTA Professional Competencies covers musical skills needed to be a successful clinician and sets music therapy apart from other expressive arts therapies. The surveyed pre-interns identified the following areas as requiring the highest levels of assistance: "knowledge of multicultural music" ( $M=$ $3.421)$, "selecting standard works for your client's needs" $(M=2.737)$, and "improvising vocally" $(M=2.52)$. The internship supervisors rated their highest levels of concern regarding "improvising vocally" $(M=3.00)$, "demonstrating basic piano skills" ( $M=2.786)$, and "leading and accompanying on guitar and piano" $(M=2.714)$.

Only two responses differed significantly between pre-interns and supervisors within the music foundations section: "demonstrating basic piano skills" $t(31)=-2.929$, $p=.006$ with an effect size of 1.049 and "leading and accompanying on guitar and piano" $U\left(N_{\text {pre-interns }}=19, N_{\text {supervisors }}=14,\right)=140.5, z=2.063, p=.046$. These strong effect sizes show that pre-interns perceive they need much less assistance to meet the competencies compared to internship supervisors' perceptions. See Table 2 for remaining statements. 
Table 1

Results for Clinical Foundations

\begin{tabular}{|c|c|c|c|c|c|}
\hline Item & $\begin{array}{l}\text { Pre- } \\
\text { intern }\end{array}$ & $\begin{array}{l}\text { Internship } \\
\text { supervisor }\end{array}$ & $t$ & $d f$ & $p$ \\
\hline & M & M & & & \\
\hline Finding out what is expected of me as an intern & 3.5263 & 2.9286 & 1.266 & 31 & .215 \\
\hline Communicating with facility staff & 2.8421 & 3.2143 & -0.920 & 31 & .365 \\
\hline Communicating with other interns/supervisors & 2.9474 & 2.5714 & 0.873 & 31 & .390 \\
\hline Organizing and managing my session space & 2.5263 & 2.8571 & -0.784 & 31 & .439 \\
\hline Maintaining client confidence & 2.5263 & 2.5000 & 0.062 & 30.341 & .951 \\
\hline Obtaining instructional resources and materials & 3.0526 & 2.6429 & 0.998 & 31 & .326 \\
\hline Planning a session & 2.2632 & 2.8571 & -1.676 & 31 & .104 \\
\hline Managing my time and work & 2.4211 & 3.2143 & -1.925 & 30.120 & .064 \\
\hline Assessing client needs & 2.9474 & 3.5714 & -1.683 & 31 & .103 \\
\hline Evaluating client's progress & 2.7368 & 3.2857 & -1.476 & 31 & .150 \\
\hline Motivating clients & 2.4737 & 2.8571 & -1.104 & 30.426 & .278 \\
\hline Dealing with individual differences among clients & 2.6842 & 2.9286 & -0.618 & 31 & .541 \\
\hline Understanding the treatment modality & 3.0000 & 2.5714 & 1.107 & 31 & .277 \\
\hline Completing administrative paperwork & 2.8421 & 2.7143 & 0.307 & 31 & .761 \\
\hline Using a variety of therapeutic methods & 2.8947 & 3.1429 & -0.660 & 29.292 & .514 \\
\hline Facilitating group discussions & 2.8947 & 3.7143 & -2.032 & 31 & .051 \\
\hline Understanding the facility's intern evaluation process & 3.5263 & 2.0000 & 4.217 & 31 & $.001^{*}$ \\
\hline $\begin{array}{l}\text { Understanding my legal rights and responsibilities as } \\
\text { a therapist }\end{array}$ & 3.1053 & 2.4286 & 1.627 & 31 & .114 \\
\hline Dealing with stress & 2.6842 & 3.5000 & -1.953 & 31 & .060 \\
\hline $\begin{array}{l}\text { Becoming aware of special services provided by facili- } \\
\text { ty }\end{array}$ & 2.7895 & 2.3571 & 1.076 & 31 & .290 \\
\hline $\begin{array}{l}\text { Closing knowledge gap between academic program } \\
\text { and internship }\end{array}$ & 3.3684 & 3.3571 & 0.031 & 31 & .976 \\
\hline
\end{tabular}

\section{Summary}

Pre-interns reported a higher need for assistance in the area of "understanding the facility's intern evaluation process" compared to internship supervisors. Pre-interns reported lower need for assistance in "demonstrating basic piano skills" and "leading and accompanying on guitar and piano" than was reported by internship supervisors.

\section{Survey 2}

The purpose of Survey 2 was to investigate internship preparedness and the differences between International and American music therapy interns and professionals. The survey was sent to a total of 2,673 individuals. A total of 465 individuals self-selected to complete the survey. Chi-square tests were used to compare the frequencies of International Interns (II), American Interns (AI), International Professionals (IP), and American Professionals (AP) as they reported the AMTA Professional Competencies they felt most and least competent in, as well as their perceived strengths and concerns when entering their internship. Two respondents did not meet the eligibility requirements, so their data were excluded from analyses. Of the respondents, participants were orga- 
Table 2

Results for Music Foundations

\begin{tabular}{|c|c|c|c|c|c|}
\hline Item & $\begin{array}{l}\text { Pre- } \\
\text { intern }\end{array}$ & $\begin{array}{l}\text { Internship } \\
\text { supervisor }\end{array}$ & $t$ & $d f$ & $p$ \\
\hline & $M$ & $M$ & & & \\
\hline Selecting standard works for your client's needs & 2.7368 & 2.5714 & 0.469 & 31 & .643 \\
\hline Transposing simple compositions & 1.8421 & 2.1429 & -0.715 & 31 & .480 \\
\hline Composing songs with simple accompaniment & 2.2105 & 2.4286 & -0.509 & 28.764 & .615 \\
\hline Demonstrating basic vocal skills & 1.7368 & 2.2143 & -1.137 & 31 & .264 \\
\hline Demonstrating basic guitar skills & 1.7895 & 2.2857 & -1.240 & 31 & .224 \\
\hline Demonstrating basic piano skills & 1.7368 & 2.7857 & -2.929 & 31 & $.006^{*}$ \\
\hline Demonstrating basic percussion skills & 2.0526 & 2.0714 & -0.051 & 31 & .960 \\
\hline Leading and accompanying on guitar and piano & 2.0526 & 2.7143 & -1.748 & 31 & .090 \\
\hline $\begin{array}{l}\text { Playing and singing basic rep of traditional, folk, and } \\
\text { pop songs by memory }\end{array}$ & 2.1579 & 2.0714 & 0.194 & 31 & .847 \\
\hline Singing in tune & 1.7368 & 1.7857 & -0.114 & 31 & .910 \\
\hline $\begin{array}{l}\text { Harmonizing and transposing simple compositions in } \\
\text { several keys }\end{array}$ & 2.0000 & 2.5714 & -1.476 & 31 & .510 \\
\hline Tuning stringed instruments & 1.4737 & 1.5000 & -0.076 & 31 & .940 \\
\hline Improvising on instruments & 2.1579 & 2.5714 & -0.874 & 31 & .389 \\
\hline Improvising vocally & 2.5263 & 3.0000 & -0.977 & 31 & .336 \\
\hline Knowledge of multicultural music & 3.4211 & 2.6429 & 1.817 & 31 & .079 \\
\hline
\end{tabular}
${ }^{*} p<.05$.

nized into four groups: 50 American Interns (AI, 10.75\%), 353 American Professionals (AP, 75.91\%), 12 International Interns (II, 2.58\%), and 50 International Professionals (IP, 10.75\%). Respondents in this survey included 399 females (86.18\%), 62 males (13.39\%), two participants identified as "other" $(0.43 \%)$, and two individuals did not respond to this question. Two hundred and thirty-seven of the respondents had earned or were pursuing a bachelor's degree $(51.18 \%), 219$ had earned or were pursuing a master's degree $(47.3 \%)$, and seven had earned or were pursuing a doctoral degree (1.51\%). A total of 416 respondents reported their first language was English (90.24\%) with Chinese being the second highest reported (5.86\%). Four hundred and thirty-nine of the participants were between the ages of $20-40$ (94.38\%), while 26 were over the age of $40(5.62 \%)$.

\section{Perceived Top Five Areas of Strength in AMTA Professional Competencies}

Participants rated which five AMTA Professional Competencies they were most proficient in prior to the start of their internship. Of the 464 participants who responded to this question, the five most reported responses were "voice skills" $(52.8 \%, n=245)$, "therapeutic relationship" (47.8\%, $n=222)$, "guitar skills" $(35.1 \%, n=163)$, "music theory and history" $(33.2 \%, n=154)$, and "major performance medium skills" $(30.2 \%, n=140)$. Table 3 indicates the top five most competent areas at the onset of internship by group association.

There were no significant associations across categorical groups (i.e., AI, II, AP, and IP) in their top five most reported proficient AMTA Professional Competencies prior to internship: "voice skills" $X^{2}(3)=5.588, p=.133$, "therapeutic relationship" $X^{2}(3)=$ $1.036, p=.792$, "guitar skills" $X^{2}(3)=4.965, p=.174$, "music theory and history" 
Table 3

Frequency Table of Perceived Top Five Most Competent AMTA Competencies

\begin{tabular}{|c|c|c|c|c|}
\hline \multirow[t]{2}{*}{ AMTA Competencies } & \multicolumn{4}{|c|}{ Percent of Cases } \\
\hline & $\begin{array}{c}\text { II } \\
n=12\end{array}$ & $\begin{array}{c}\text { Al } \\
n=49\end{array}$ & $\begin{array}{c}\text { IP } \\
n=50\end{array}$ & $\begin{array}{c}\text { AP } \\
n=353\end{array}$ \\
\hline Music theory and history & $58.3 \%$ & $28.6 \%$ & $30.0 \%$ & $33.4 \%$ \\
\hline Composition and arranging skills & $16.7 \%$ & $4.1 \%$ & $12.0 \%$ & $9.1 \%$ \\
\hline Major performance medium skills & $41.7 \%$ & $30.6 \%$ & $26.0 \%$ & $30.3 \%$ \\
\hline Keyboard skills & $75.0 \%$ & $22.4 \%$ & $42.0 \%$ & $25.2 \%$ \\
\hline Voice skills & $66.7 \%$ & $55.1 \%$ & $38.0 \%$ & $54.1 \%$ \\
\hline Guitar skills & $25.0 \%$ & $46.9 \%$ & $26.0 \%$ & $35.1 \%$ \\
\hline Percussion skills & $16.7 \%$ & $8.2 \%$ & $18.0 \%$ & $11.0 \%$ \\
\hline Non-symphonic instrumental skills & $0 \%$ & $4.1 \%$ & $6.0 \%$ & $3.1 \%$ \\
\hline Improvisation skills & $16.7 \%$ & $12.2 \%$ & $32.0 \%$ & $15.6 \%$ \\
\hline Conducting skills & $0 \%$ & $12.2 \%$ & $4.0 \%$ & $4.2 \%$ \\
\hline Movement skills & $0 \%$ & $10.2 \%$ & $6.0 \%$ & $9.9 \%$ \\
\hline Therapeutic applications & $8.3 \%$ & $22.4 \%$ & $30.0 \%$ & $23.5 \%$ \\
\hline Therapeutic principles & $25.0 \%$ & $18.4 \%$ & $24.0 \%$ & $23.8 \%$ \\
\hline Therapeutic relationship & $58.3 \%$ & $53.1 \%$ & $46.0 \%$ & $47.0 \%$ \\
\hline Foundations and principles & $8.3 \%$ & $20.4 \%$ & $24.0 \%$ & $24.6 \%$ \\
\hline Client assessment & $8.3 \%$ & $16.3 \%$ & $8.0 \%$ & $8.2 \%$ \\
\hline Treatment planning & $33.3 \%$ & $22.4 \%$ & $14.0 \%$ & $21.2 \%$ \\
\hline Therapy implementation & $8.3 \%$ & $16.3 \%$ & $12.0 \%$ & $18.7 \%$ \\
\hline Therapy evaluation & $8.3 \%$ & $4.1 \%$ & $0 \%$ & $2.5 \%$ \\
\hline Documentation & $8.3 \%$ & $32.7 \%$ & $16.0 \%$ & $31.2 \%$ \\
\hline Termination/discharge planning & $0 \%$ & $0 \%$ & $0 \%$ & $1.1 \%$ \\
\hline Professional role/ethics & $25.0 \%$ & $32.7 \%$ & $20.0 \%$ & $31.2 \%$ \\
\hline Interprofessional collaboration & $0 \%$ & $10.2 \%$ & $12.0 \%$ & $7.9 \%$ \\
\hline Supervision and administration & $0 \%$ & $2.0 \%$ & $0 \%$ & $2.3 \%$ \\
\hline Research methods & $0 \%$ & $8.2 \%$ & $8.0 \%$ & $14.2 \%$ \\
\hline Other & $0 \%$ & $0 \%$ & $2.0 \%$ & $1.1 \%$ \\
\hline
\end{tabular}

Note. Top five responses for each subgroup are highlighted in bold, light grey.

$X^{2}(3)=4.271, p=.234$, and "major performance medium skills" $X^{2}(3)=1.170, p$ $=.760$.

\section{Perceived Top Five Areas of Weakness in AMTA Competencies}

Of the 460 participants who responded to this question, the five most reported areas which needed improvement were "improvisation skills" $(48.3 \%, n=222)$, "client assessment" (46.7\%, $n=250)$, "guitar skills" $(41.5 \%, n=191)$, "therapeutic applications" (38.0\%, $n=175)$, and "therapy implementation" (37.0\%, $n=170)$. Table 4 indicates the top five reported areas needing improvement at the onset of internship by group association.

Chi-square analyses indicated there were no significant associations across categorical groups in the top five most reported areas of improvement: "improvisation skills" $X^{2}(3)=3.702, p=.295$, "client assessment" $X^{2}(3)=4.133, p=.247$, "guitar skills" 
Table 4

Frequency Table of Perceived Five Areas Needing Improvement in AMTA Competencies

\begin{tabular}{|c|c|c|c|c|}
\hline \multirow[t]{2}{*}{ AMTA Competencies } & \multicolumn{4}{|c|}{ Percent of Cases } \\
\hline & $\begin{array}{c}\text { II } \\
n=12\end{array}$ & $\begin{array}{c}\text { Al } \\
n=49\end{array}$ & $\begin{array}{c}\text { IP } \\
n=49\end{array}$ & $\begin{array}{c}\text { AP } \\
n=350\end{array}$ \\
\hline Music theory and history & $0 \%$ & $6.1 \%$ & $4.1 \%$ & $2.0 \%$ \\
\hline Composition and arranging skills & $0 \%$ & $12.2 \%$ & $12.2 \%$ & $13.7 \%$ \\
\hline Major performance medium skills & $0 \%$ & $0 \%$ & $0 \%$ & $0.6 \%$ \\
\hline Keyboard skills & $8.3 \%$ & $42.9 \%$ & $20.4 \%$ & $32.0 \%$ \\
\hline Voice skills & $8.3 \%$ & $20.4 \%$ & $18.4 \%$ & $19.4 \%$ \\
\hline Guitar skills & $50.0 \%$ & $36.7 \%$ & $34.7 \%$ & $42.9 \%$ \\
\hline Percussion skills & $0 \%$ & $6.1 \%$ & $8.2 \%$ & $12.0 \%$ \\
\hline Non-symphonic instrumental skills & $0 \%$ & $2.0 \%$ & $4.1 \%$ & $1.7 \%$ \\
\hline Improvisation skills & $50.0 \%$ & $44.9 \%$ & $36.7 \%$ & $50.3 \%$ \\
\hline Conducting skills & $16.7 \%$ & $0 \%$ & $2.0 \%$ & $1.7 \%$ \\
\hline Movement skills & $8.3 \%$ & $6.1 \%$ & $2.0 \%$ & $6.6 \%$ \\
\hline Therapeutic applications & $50.0 \%$ & $42.9 \%$ & $36.7 \%$ & $37.1 \%$ \\
\hline Therapeutic principles & $50.0 \%$ & $18.4 \%$ & $16.3 \%$ & $8.0 \%$ \\
\hline Therapeutic relationship & $33.3 \%$ & $16.3 \%$ & $18.4 \%$ & $18.0 \%$ \\
\hline Foundations and principles & $8.3 \%$ & $12.2 \%$ & $10.2 \%$ & $3.7 \%$ \\
\hline Client assessment & $41.7 \%$ & $36.7 \%$ & $57.1 \%$ & $46.9 \%$ \\
\hline Treatment planning & $25.0 \%$ & $26.5 \%$ & $36.7 \%$ & $34.6 \%$ \\
\hline Therapy implementation & $25.0 \%$ & $32.7 \%$ & $44.9 \%$ & $36.9 \%$ \\
\hline Therapy evaluation & $16.7 \%$ & $14.3 \%$ & $24.5 \%$ & $15.4 \%$ \\
\hline Documentation & $50.0 \%$ & $28.6 \%$ & $36.7 \%$ & $32.9 \%$ \\
\hline Termination/discharge planning & $25.0 \%$ & $18.4 \%$ & $14.3 \%$ & $20.6 \%$ \\
\hline Professional role/ethics & $0 \%$ & $6.1 \%$ & $12.2 \%$ & $11.1 \%$ \\
\hline Interprofessional collaboration & $16.7 \%$ & $38.8 \%$ & $28.6 \%$ & $32.0 \%$ \\
\hline Supervision and administration & $8.3 \%$ & $12.2 \%$ & $8.2 \%$ & $6.6 \%$ \\
\hline Research methods & $8.3 \%$ & $12.2 \%$ & $2.0 \%$ & $8.3 \%$ \\
\hline Other & $0 \%$ & $0 \%$ & $0 \%$ & $0.6 \%$ \\
\hline
\end{tabular}

Note. Top five responses for each subgroup are highlighted in bold, light grey.

$X^{2}(3)=2.254, p=.521$, "therapeutic applications" $X^{2}(3)=1.343, p=.719$, and "therapy implementation" $X^{2}(3)=2.333, p=.506$.

\section{Perceived Top Three Strengths Entering Internship (Not Related to AMTA Professional Competencies)}

Results for this question were calculated from 454 total participants. The most frequently reported were "general aptitude" $(48.7 \%, n=221)$, "professionalism" (46.7\%, $n=212)$, and "general preparation/being prepared" $(37.7 \%, n=171)$. Table 5 includes the remaining statements across all group associations.

Chi-square analyses indicated there were no significant associations across categorical groups in the top three strengths when entering internships: "general aptitude" $X^{2}(3)=4.657, p=.199$, "professionalism" $X^{2}(3)=2.805, p=.423$, and "general preparation/being prepared" $X^{2}(3)=1.939, p=.585$. 
Table 5

Frequency Table of Perceived Top Three Strengths Entering Internship (Not AMTA Competency Related)

\begin{tabular}{|c|c|c|c|c|}
\hline \multirow[t]{2}{*}{ Categories } & \multicolumn{4}{|c|}{ Percent of Cases } \\
\hline & $\begin{array}{c}\mathrm{II} \\
n=12\end{array}$ & $\begin{array}{c}\text { Al } \\
n=49\end{array}$ & $\begin{array}{c}\text { IP } \\
n=48\end{array}$ & $\begin{array}{c}\text { AP } \\
n=345\end{array}$ \\
\hline $\begin{array}{l}\text { General preparation/being prepared (or having } \\
\text { knowledge) }\end{array}$ & $50.0 \%$ & $30.6 \%$ & $39.6 \%$ & $38.0 \%$ \\
\hline $\begin{array}{l}\text { General aptitude (how well suited you are to be a } \\
\text { therapist) }\end{array}$ & $50.0 \%$ & $34.7 \%$ & $56.2 \%$ & $49.6 \%$ \\
\hline Enthusiasm for supervisor/placement & $25.0 \%$ & $32.7 \%$ & $27.1 \%$ & $33.0 \%$ \\
\hline $\begin{array}{l}\text { Physical environment (Having enough money, mov- } \\
\text { ing to a new location, etc.) }\end{array}$ & $8.3 \%$ & $10.2 \%$ & $12.5 \%$ & $7.2 \%$ \\
\hline Anticipated response from clients & $0 \%$ & $12.2 \%$ & $8.3 \%$ & $2.9 \%$ \\
\hline Your competence as a music therapy intern & $41.7 \%$ & $18.4 \%$ & $22.9 \%$ & $15.7 \%$ \\
\hline $\begin{array}{l}\text { Specific preparation/specific content knowledge } \\
\text { related to internship population(s) }\end{array}$ & $0 \%$ & $14.3 \%$ & $12.5 \%$ & $12.2 \%$ \\
\hline Discipline & $16.7 \%$ & $12.2 \%$ & $20.8 \%$ & $18.3 \%$ \\
\hline $\begin{array}{l}\text { Your ability to apply the knowledge you developed } \\
\text { pre-internship }\end{array}$ & $41.7 \%$ & $28.6 \%$ & $25.0 \%$ & $26.4 \%$ \\
\hline Being respected & $8.3 \%$ & $6.1 \%$ & $6.2 \%$ & $2.0 \%$ \\
\hline Meeting expectations & $0 \%$ & $4.1 \%$ & $6.2 \%$ & $11.9 \%$ \\
\hline Your professionalism & $41.7 \%$ & $53.1 \%$ & $37.5 \%$ & $47.2 \%$ \\
\hline Communicating effectively & $16.7 \%$ & $30.6 \%$ & $18.8 \%$ & $22.0 \%$ \\
\hline Using time effectively & $0 \%$ & $10.2 \%$ & $6.2 \%$ & $12.8 \%$ \\
\hline Other & $0 \%$ & $0 \%$ & $0 \%$ & $0.9 \%$ \\
\hline
\end{tabular}

Note. Top three responses for each subgroup are highlighted in bold, light grey.

\section{Perceived Top Three Concerns Entering Internship (Not AMTA Competency Related)}

Results for this question were calculated from 452 total participants. Frequency counts determined the primary concerns in relation to internship preparedness included "physical environment concerns (money, moving, etc.)" $(42.5 \%, n=192)$ and "specific preparation/lack of specific content knowledge" $(34.5 \%, n=156)$, followed by "expectations (not meeting)" $(27.4 \%, n=124)$. See Table 6 for remaining statements.

Chi-square analyses indicated there was a significant association across categorical groups in the top three areas of concerns/worries of "physical environment concern," $X^{2}(3)=14.838, p<.005$, "clients not responding," $X^{2}(3)=9.348, p<.05$, and "not being professional," $X^{2}(3)=18.259, p<.001$.

\section{Differences Between American and International Participants}

Analyses were also computed across statements outside of the top items that were reported. Chi-square analyses were used to indicate whether or not a relationship existed across the categorical variables. Significant associations within the proficient AMTA Professional Competencies question included "keyboard skills," $X^{2}(3)=20.282, p<$ .001 and "improvisation skills," $X^{2}(3)=9.369, p<.05$. There were four areas of significant associations across groups in perceived least proficient AMTA competencies. These included "keyboard skills," $X^{2}(3)=8.631, p<.05$, "conducting skills," $X^{2}(3)$ $=14.811, p<.01$, "therapeutic principles," $X^{2}(3)=25.883, p<.001$, and "foundations and principles" $X^{2}(3)=8.610, p<.05$. 
Table 6

Frequency Table of Perceived Top Three Concerns Entering Internship (Not AMTA Competency Related)

\begin{tabular}{|c|c|c|c|c|}
\hline \multirow[t]{2}{*}{ Categories } & \multicolumn{4}{|c|}{ Percent of Cases } \\
\hline & $\begin{array}{c}\text { II } \\
n=12\end{array}$ & $\begin{array}{c}\text { Al } \\
n=49\end{array}$ & $\begin{array}{c}\text { IP } \\
n=48\end{array}$ & $\begin{array}{c}\text { AP } \\
n=343\end{array}$ \\
\hline $\begin{array}{l}\text { General preparation/being prepared (or not having } \\
\text { knowledge) }\end{array}$ & $8.3 \%$ & $28.6 \%$ & $20.8 \%$ & $19.5 \%$ \\
\hline Failure (not cut out for therapy) & $16.7 \%$ & $18.4 \%$ & $14.6 \%$ & $23.0 \%$ \\
\hline Concerns about supervisor/placement & $25.0 \%$ & $16.3 \%$ & $12.5 \%$ & $16.3 \%$ \\
\hline $\begin{array}{l}\text { Physical environment concerns (money, moving, } \\
\text { etc.) }\end{array}$ & $8.3 \%$ & $59.2 \%$ & $29.2 \%$ & $43.1 \%$ \\
\hline Clients not responding & $58.3 \%$ & $16.3 \%$ & $25.0 \%$ & $25.7 \%$ \\
\hline Competent & $0 \%$ & $16.3 \%$ & $22.9 \%$ & $15.7 \%$ \\
\hline Specific preparation/lack of content knowledge & $33.3 \%$ & $34.7 \%$ & $45.8 \%$ & $32.9 \%$ \\
\hline Discipline & $8.3 \%$ & $6.1 \%$ & $2.1 \%$ & $2.0 \%$ \\
\hline Not being able to apply knowledge & $33.3 \%$ & $16.3 \%$ & $22.9 \%$ & $16.0 \%$ \\
\hline Not being respected & $0 \%$ & $10.2 \%$ & $14.6 \%$ & $11.7 \%$ \\
\hline Expectations (not meeting) & $8.3 \%$ & $36.7 \%$ & $18.8 \%$ & $28.0 \%$ \\
\hline Not being professional & $8.3 \%$ & $2.0 \%$ & $14.6 \%$ & $2.3 \%$ \\
\hline Embarrassing (saying something inappropriate) & $41.7 \%$ & $10.2 \%$ & $14.6 \%$ & $14.6 \%$ \\
\hline Clients will not like me & $16.7 \%$ & $4.1 \%$ & $6.2 \%$ & $7.9 \%$ \\
\hline Using time effectively & $25.0 \%$ & $14.3 \%$ & $14.6 \%$ & $25.4 \%$ \\
\hline Other & $8.3 \%$ & $6.1 \%$ & $18.8 \%$ & $9.3 \%$ \\
\hline
\end{tabular}

Note. Top three responses for each subgroup are highlighted in bold, light grey.

Within the perceived strengths questions, there was a significant association across categorical groups in "anticipated response from clients," $X^{2}(3)=10.626, p<.05$. Finally, there were significant associations in "physical environment (having enough money, moving to a new location, etc.)," $X^{2}(3)=14.838, p<.05$, "clients not responding," $X^{2}(3)$ 9.348, $p<.05$, "not being professional," $X^{2}(3)=18.259, p<.001$, and "embarrassing (saying something inappropriate)," $X^{2}(3)=8.042, p<.05$ within the top areas of concern.

\section{Factors in Choosing an Internship}

Survey 2 also asked respondents what was important to them when choosing an internship. The top three responses when aggregating all participants in deciding where to complete their internship training included: (1) clinical population $(91.8 \%, n=423)$; (2) location (i.e., city, state), $(68.5 \%, n=316)$; and (3) opportunities to work with other disciplines/professions $(37.5 \%, n=173)$. AI $(n=50)$, AP $(n=350)$, and IP $(n=49)$ all chose the same top three factors as listed above. However, II chose (1) clinical population (83.3\%, $n=10$ ); (2) opportunities to work with disciplines/professions $(58.3 \%, n=7)$; and (3) supervisor $(50 \%, n=6)$ as their top three factors when choosing an internship.

\section{Expectations Met in Internship}

To compare the differences between subgroups regarding if expectations were met in their internship, a nonparametric test was completed using the Kruskal-Wallis test. A total of eight areas were significantly different between subgroups. Statements included: "major performance medium" $(H(3)=8.16, p=.043)$, "keyboard skills" $(H(3)=$ 
Table 7

Kruskal-Wallis Results on Expectations Met in Internship

\begin{tabular}{|c|c|c|c|c|}
\hline AMTA Competencies & $N$ & $d f$ & $H$ & $p$ \\
\hline Music theory and history & 435 & 3 & 5.950 & .114 \\
\hline Composition and arranging skills & 435 & 3 & 6.570 & .087 \\
\hline Major performance medium skills & 434 & 3 & 8.160 & $.043^{*}$ \\
\hline Keyboard skills & 435 & 3 & 11.291 & $.010^{*}$ \\
\hline Voice skills & 432 & 3 & 18.731 & $.001^{*}$ \\
\hline Non-symphonic instrument skills & 432 & 3 & 3.636 & .304 \\
\hline Improvisation skills & 426 & 3 & 2.865 & .413 \\
\hline Conducting skills & 431 & 3 & 7.524 & .057 \\
\hline Movement skills & 432 & 3 & 5.778 & .123 \\
\hline Therapeutic applications & 422 & 3 & 12.690 & $.005^{*}$ \\
\hline Therapeutic principles & 435 & 3 & 7.127 & .068 \\
\hline Therapeutic relationship & 433 & 3 & 6.716 & .082 \\
\hline Foundations and principles & 434 & 3 & 6.598 & .086 \\
\hline Client assessment & 434 & 3 & 5.504 & .138 \\
\hline Treatment planning & 433 & 3 & 2.555 & .465 \\
\hline Therapy implementation & 433 & 3 & 11.652 & $.009^{*}$ \\
\hline Therapy evaluation & 432 & 3 & 12.676 & $.005^{*}$ \\
\hline Documentation & 434 & 3 & 7.270 & .064 \\
\hline Termination/discharge planning & 434 & 3 & 1.836 & .607 \\
\hline Professional role/ethics & 433 & 3 & 11.105 & $.011^{*}$ \\
\hline Interprofessional collaboration & 432 & 3 & 18.182 & $.001^{*}$ \\
\hline Supervision and administration & 433 & 3 & 4.806 & .187 \\
\hline Research methods & 433 & 3 & 5.412 & .144 \\
\hline
\end{tabular}

${ }^{*} p<.05$.

$11.291, p=.01)$, "voice skills" $(H(3)=18.731, p=0.01)$, "therapeutic applications" $(H(3)=12.690, p=.005)$, "therapy implementation" $(H(3)=11.652, p=.009)$, "therapy evaluation" $(H(3)=12.676, p=.005)$, "professional role/ethics" $(H(3)=$ $11.105, p=.011)$, and "interdisciplinary collaboration" $(H(3)=18.182, p=.001)$. Table 7 lists all statements.

A total of eight statements demonstrated a significant difference between groups. Post hoc analyses were completed using pairwise comparisons for each subgroup. For the "major performance" competency, differences were between IP (mean rank 180.18) and AI (mean rank 247.03) ( $p=.048$ ). For "keyboard skills," IP (mean rank 179.58) and II (mean rank 297.97) showed differences $(p=.021)$. "Voice skills" showed differences between IP (mean rank 152.81) and AP (mean rank 223.43) ( $p=$ $.001)$ and IP (mean rank 152.81) and AI (mean rank 223.62) $(p=.011)$. The competency of "therapeutic applications" showed differences between IP (mean rank 155.73) and AP (mean rank 217.95) ( $p=.005)$ and IP (mean rank 155.73) and AI (mean rank 221.41) $(p=.050)$. "Therapy implementation differeces existed between IP (mean rank 170.48) and AP (mean rank 221.49) ( $p=.013$ ) and IP (mean rank 170.48) and AI (mean rank 233.23) ( $p=.029)$. In "therapy evaluation" differences existed between IP (mean rank 166.41) and AP (mean rank 219.60) $(p=.018$ ) and IP (mean rank 166.41) and AI (mean rank 235.59) ( $p=.019$ ). "Professional role/ethics" showed differences between IP (mean rank 175.14) and AP (mean rank 221.00) $(p=.038)$ and IP (mean 
rank 175.14) and AI (mean rank 236.89) ( $p=.033$ ). The competency of "interdisciplinary collaboration" showed differences between IP (mean rank 153.48) and AP (mean rank 221.67) $(p=.001)$ and IP (mean rank 153.48) and AI (mean rank 243.26) $(p=$ $.001)$.

\section{Summary}

In relation to the top five AMTA Professional Competencies respondents felt most and least confident in, there were no significant differences across the four groups. There were also no significant differences in respondents' top three perceived strengths when entering internship. There were, however, differences in respondents' top three concerns when entering internship in terms of preparedness relating to "physical environment concern," "clients not responding," and "not being professional." There were also significant differences across groups outside of the top five responses. A Kruskal-Wallis test was used to compare which expectations were met in internship between groups. Items that significantly differed across groups included "major performance medium," "keyboard skills," "voice skills," "therapeutic applications," "therapy implementation," "therapy evaluation," "professional role/ethics," and "interdisciplinary collaboration."

\section{Survey 3}

While Surveys 1 and 2 examined specific elements of clinical training, Survey 3 sought to understand participants' overall satisfaction with their academic curriculum and internship. The survey was sent to a total of 7,183 MT-BC's and 777 participants self-selected to complete a survey that explored the satisfaction of music therapy professionals with music therapy courses and programs. The respondents included 210 from the Great Lakes Region (27.03\%), 183 from the Mid-Atlantic Region (23.55\%), 125 from the Southeastern Region (16.09\%), 95 from the Midwest Region (12.23\%), 64 from the Western Region (8.24\%), 58 from the Southwestern Region (7.46\%), and 42 from the New England Region (5.41\%) of AMTA. Respondents reported graduation years ranging from 1971-2017. A total of $50.84 \%$ of the respondents graduated between 2011-2017 $(n=395)$.

\section{Satisfaction with Training}

Overall, $92.72 \%$ ( $n=688$ ) of respondents were satisfied with their courses, $92.73 \%$ ( $n=689$ ) were satisfied with the quality of teaching in their program, and $88.46 \%$ ( $n$ $=664$ ) were satisfied with their clinical practicum coursework. However, $11.54 \%$ ( $n$ $=84$ ) of respondents reported their clinical practicum coursework was not sufficient. In terms of practicum experience, $90.01 \%(n=658)$ were satisfied with the range of practicum experiences offered.

Individuals were largely satisfied with their overall preparation for professional practice with $92.88 \%(n=587)$ feeling satisfied with their undergraduate training. A chi-square test was used to analyze the relationship of responses regarding the overall satisfaction level with the undergraduate or equivalency curriculum and the satisfaction level related to the relevancy of the courses. Results indicated that a relationship existed between the satisfaction levels of overall curriculum and relevance of the courses, $X^{2}(25, N=587)=823.583, p<.001$. In addition, a chi-square test indicated that a relationship existed between satisfaction level with curriculum and the quality of teaching, $X^{2}(25, N=587)=672.611, p<.001$.

A Spearman correlation test was used for three analyses: 1) graduation year and the satisfaction level of the relevance of courses; 2) graduation year and the satisfaction level of the quality of teaching; and 3) graduation year and the overall satisfaction level of undergraduate or equivalency curriculum. There was no significant relationship between graduation year and the satisfaction level of the relevance of courses $\left(r_{\mathrm{s}}=\right.$ $-.041, p<.135$ ). Also, there was no significant relationship between graduation year and the satisfaction level of the quality of teaching $\left(r_{\mathrm{s}}=.038, p<.151\right)$. However, 
there was a significant relationship between graduation years and the overall satisfaction level of the undergraduate/graduate equivalency curriculum $\left(r_{\mathrm{s}}=.070, p<\right.$ .040). The music therapists who graduated more recently had a higher satisfaction level, though it is important to mention the effect size is small.

\section{Overall Satisfaction}

The responses showed that $78.58 \%(n=491)$ of professionals would choose the same university/college music therapy program again. However, $20.42 \%(n=126)$ indicated they would choose a different music therapy program. The results also showed that $76.26 \%(n=453)$ would choose music therapy again, or, if possible, they would double major with another discipline during their undergraduate training or earn a higher degree in another discipline. However, $23.74 \%(n=141)$ of respondents specified they would choose another profession. Responses included: occupational therapy ( $n=$ 28); psychology $(n=25)$; social work $(n=20)$; and speech language pathology ( $n$ $=16$ ). According to the responses, the most common reasons respondents indicated a desire to choose another profession were related to low income $(n=24)$, limited job availability $(n=24)$, burn out $(n=4)$, and constant advocacy efforts $(n=3)$.

\section{Summary}

Broadly, respondents were satisfied with their clinical training and preparation for professional practice. However, there were still some who reported that their training was insufficient or that they would choose a different academic program or a different field entirely.

\section{Discussion}

\section{Survey 1}

This study examined differences in perception between pre-interns and internship supervisors in regard to clinical and music foundations according to the AMTA Professional Competencies. This study replicated questions from the Knight (2008) study with some additional questions regarding music foundations. Areas of difference between the two studies are explained below.

In other studies that surveyed students' pre-internship concerns, results showed that the top concerns of most students were musical skills (Wheeler, 2002), general preparation for an internship or not having knowledge (Madsen \& Kaiser, 1999), and knowing what is expected of them as an intern (Knight, 2008). The musical skills concern is mirrored in the internship supervisors (Jenkins, 2013) with the addition of other concerns for their interns. Internship supervisors and directors identified "making sure competencies were met" as the most difficult aspect of supervision (Tanguay, 2008). The results of this study fall in line with the results of previous studies and reinforce some areas of concern that can be addressed in academic programs.

The pre-interns reported a higher need for "understanding the facility's intern evaluation process" compared to the internship supervisors. This area cannot truly be addressed in the academic program due to each facility having a different evaluation process. The responsibility of addressing this concern for assistance lies solely with the internship supervisor and can only be addressed once the internship has begun.

Pre-interns reported a lower concern for "demonstrating basic piano skills" compared to internship supervisors. Additionally, there has been a reduction in the perceived importance of piano skills in music therapy internship supervisors in the last 25 years (Jenkins, 2013). A survey of 92 internship directors showed that piano skills are now ranked third behind vocal skills and guitar skills (Jenkins, 2013), whereas a similar study by Brookins (1984) ranked piano skills as the most essential (Jenkins, 2013; Brookins, 1984). The reduction of importance in piano could be tied to the portability of the guitar and the ease in which a session can be led from a guitar rather than be- 
hind a piano. More experienced internship supervisors may place a higher importance on piano skills and individual clinical preferences may sway the outcome of internship supervisors.

\section{Limitations}

A limitation of this study is that this survey only targeted the Great Lakes Region and was not available nationally.

\section{Survey 2}

This survey examined differences in perceptions of clinical preparedness in relation to the AMTA Professional Competencies, personal strengths and concerns prior to internship, factors in choosing an internship as well as satisfaction with clinical training, and whether there were differences based on cultural demographics. Some significant differences were found in the responses between American and International respondents.

\section{Perceived Preparation Related to AMTA Professional Competencies}

International participants reported "documentation" as one of their top five skills that they wanted to improve during their internships while American participants chose it as an area in which they felt competent. Since documentation is typically in English, American participants may be comfortable completing paperwork in their first language whereas the language barrier makes paperwork more difficult for International participants.

A higher percentage of American participants chose "voice skills," while a higher percentage of International participants chose "piano skills" as competencies they felt confident in prior to internship.

Two points of particular interest to academic programs and internship sites: 1) "Termination/discharge planning" was least selected for all groups as a competency in which they felt confident, 2) "Guitar skills" was selected by American participants as a competency they felt most confident about, but it was also a competency they most wanted to improve during internship. This could indicate that there is a wide range of needs concerning students' training on guitar.

\section{Perceived Top Three Strengths and Concerns Entering Internship (Not Related to AMTA Professional Competencies)}

"Physical environment (money, moving, etc.)" was in the top three choices for all groups. Internships are often unpaid and require students to move to unfamiliar places. Participants who chose "other" mentioned concerns about financial instability, their inability to work full-time during internship, and working in a new city. International interns also chose "embarrassing (saying something inappropriate)" as a concern. For Asian students especially, their cultures are very different in terms of communication, social practice, and belief systems. Therefore, International interns may have more worries about communicating something inappropriate.

\section{Factors in Choosing an Internship}

"Client population" was a top choice for all four groups. "Location" was the second highest factor for American interns, American professionals, and International professionals. Additional comments were similar to those in the Top Three Concerns question, citing mainly concerns about the ability to work part-time while in internship to combat financial instability. Another top factor in choosing an internship was "opportunities to work with other disciplines/professions." This may be notable for internship sites as it indicates that students have a strong interest in interprofessional collaboration. 
It was surprising that "start date" was not chosen as a top factor for International participants. International students in music therapy typically hold F1 visas, which require that the student be enrolled in a certain number of credits. This means that they need to be enrolled in other courses during their internship, making the start and end dates of their internship very important.

\section{Expectations Met in Internships}

Over $91 \%$ of participants indicated their expectations were mostly or completely met during their internship in the following AMTA Competencies: "therapeutic relationship," "professional role/ethics," "therapy implementation," "documentation," and "voice skills." This supports findings from the Clements-Cortes (2019) mixed-methods study on the importance of a clinical internship in providing valued experience and supporting improvement in clinical, musical, and personal skills. In the ClementsCortes study, the significant areas needing improvement from pre- to post-internship that align with the present study include "current vocal skills when singing in a music therapy session," "level of writing music therapy treatment summaries/case studies," and "level of completing music therapy treatment plans" (Clements-Cortes, 2019). This study expanded on this information by comparing the weighted scores of expectation evaluations of the AMTA Competencies between American groups and International groups, finding that weighted scores were higher in the American groups. This may indicate that American interns' expectations were met to a higher degree than those of International interns due to higher demands placed on International interns as they encounter more obstacles in learning and clinical training.

\section{Limitations}

There are several limitations for Survey 2: 1) differing group sizes affected betweengroup comparisons, 2) respondents could have misunderstood some questions due to lack of details/specificity (i.e., more than 10 respondents skipped rating the category "exceptionality," possibly because of confusion as to what the term was referring to, and 3) individuals within the International groups still have diverse experiences related to their cultural backgrounds that could have caused heterogeneity within the group.

\section{Survey 3}

This portion of the present study looked at professional music therapists' satisfaction with their undergraduate experience. Based on the survey results, most professional music therapists were satisfied with their undergraduate or equivalency curriculum training in regard to the quality of teaching, course relevance, pre-internship clinical practicum settings, and overall training experience.

Most respondents were satisfied with their pre-internship clinical training. However, when compared to a similar study by Hodgetts et al. (2007) in the field of occupational therapy, the percentage of music therapy students satisfied with the teaching quality was lower $(92.73 \%, n=689)$ than occupational therapy $(98.77 \%, n=241)$. The percentage of respondents satisfied with their training $(92.88 \%, n=587)$ was also lower than occupational therapy $(97.95 \%, n=239)$. This could be due to a smaller sample size as well as a smaller range of graduation years in the Hodgetts et al. (2007) study. A larger range of graduation years allows for more variation in the quality of curriculum and ability to remember undergraduate training. Additionally, the survey by Hodgetts et al. (2007) was limited to a single university.

Many factors may contribute to the number of respondents who reported they would choose a different profession instead of music therapy $(23.74 \%, n=141)$. Approximately the same number of respondents indicated they would not choose the same university/college's music therapy program $(20.42 \%, n=126)$. Among other things, this could be attributed to the fact that academic programs have different the- 
oretical orientations, and a student may find they have a different preference in their professional life. Dissatisfaction may have less to do with the music therapy program itself and more to do with the university as a whole. Additionally, if some students are underprepared when they enter the field, they may find their work more difficult and less enjoyable and rewarding. Another reason as to why individuals may want to choose another profession could be the average salary related to years in the profession (AMTA Member Survey and Workforce Analysis, 2018).

\section{Limitations}

Since the extant literature on music therapy curriculum research is limited and outdated, current parallels could only be drawn between other professions. However, this is limited by the difference in content. Thus, deep comparisons between early studies, current studies, and other professions are limited.

\section{Conclusions}

This study provides information on the experiences of music therapy students before, during, and after the completion of their AMTA internships. It highlights how intern supervisors perceive the preparedness and development of their interns based on the AMTA Professional Competencies. A unique aspect of this research is comparisons across American and International interns and professionals, shedding light on how International interns and professionals may have different experiences within the process of completing or supervising music therapy internships in America. Overall, those surveyed were satisfied with their training and coursework. Future research might follow up with students and supervisors after internship experiences have been completed to determine what areas they improved in and how prepared interns truly are to enter the professional world. The internship is a crucial experience for the developing music therapist, as experiences from practicum and knowledge taken from coursework are applied in increased depth in a new setting and under new supervision. The level of preparedness for this experience, combined with supervision that helps interns to develop in areas of need, sets the foundation for effective clinical work in this rapidly developing field.

\section{About the Authors}

Edward Roth: Edward Roth, PhD, MT-BC currently serves as Professor and Director of Music Therapy and Director Brain Research and Interdisciplinary Neurosciences (BRAIN) laboratory, both at Western Michigan University. His research has addressed the nexus of music and neuroscience by studying the neurobiology and clinical application of music improvisation and how improvisation creates experiences of flow, creative self-expression, and social bonding for people with issues related to mental health.

He has worked with clients diagnosed with various neurological, physical, and mental health disorders. He completed his clinical internship training in adult and adolescent psychiatry at the University of Michigan Medical Center (Ann Arbor, MI) and while a graduate student at Colorado State University, he co-facilitated music therapy and counseling groups for adolescents diagnosed with post-traumatic stress and acute anxiety from Columbine High School (Littleton, CO). His clinical work focused on children and teenagers with neurologic disorders, particularly traumatic brain injuries. Contact: edward.roth@wmich.edu

Xueyan Hua: Xueyan is from China and has lived in the US since 2010. She completed her bachelor's degree and master's degree in music therapy at Western Michigan University. Xueyan published a case study for the book "Music Therapy for Multisensory and Body Awareness in Children and Adult with Severe to Profound Multiple Disabilities" by Roberta S. Adler and Olga V. Samsonova-Jellison. Currently, she is working on her MBA degree at Western Michigan University, at the same time 
she is mainly taking care her little baby and doing some clinical practices. Contact: xueyan.hua@wmich.edu

Wang Lu: Wang Lu is a music therapist and the founder of music therapy and recreation programs at Beijing Taikang Yanyuan Rehab Hospital in China. Wang loves popularizing knowledge of music therapy. Since 2016, she runs a music therapy official account through WeChat with her schoolmates for professionals and others who are interested in music therapy field in China. After graduating from Western Michigan University, she worked with some Chinese pioneer music therapists, and published Music Therapy-Melody for Wellness by People's Medical Publishing House, which is the top medical press in China. Contact: 253048715@qq.com

Jordan Blitz Novak: Jordan Blitz Novak, MM, MT-BC is the founder and owner of The Great Lakes Music Company, a community-centered music therapy private practice in Northwest Indiana that serves over 200 individuals. She completed her undergraduate degree in music therapy in 2011 from Illinois State University and completed her master's in music therapy from Western Michigan University in 2016. Jordan has been a practicing music therapist since 2012, specializing in autism spectrum disorder and intellectual disabilities. She presented at the GLR regional conference in 2018 on music therapy and ABA collaboration. Jordan plans to continue her efforts in advancing music therapy education and training by supporting students, interns, and professionals to have meaningful and long careers. Contact: jnovak@theglmc.com

Fei Wang: Fei Wang graduated from Western Michigan University with a master's degree in Music Therapy. Fei Wang is a teacher who works at Wuhan Conservatory of Music teaching music therapy courses, works as a psychological consultant, and supervises students' clinical practicum. Fei Wang has 7 years of clinical experience, she worked at the hospital, nursing home, and special education school with different kinds of clients. Contact: feiwang900706@126.com

Taylorlyn N. Mehnert: Taylorlyn (she/her) completed her bachelor's degree and her master's degree in music therapy at Western Michigan University (WMU). She completed her internship in San Diego at MusicWorx Inc following her undergraduate degree. Taylorlyn worked as a research assistant in the Brain Research and Interdisciplinary Neurosciences (BRAIN) Lab affiliated with WMU under the direction of Alycia Sterenberg Mahon and Edward Roth. Contact: taylorlyn.n.mehnert@wmich.edu

Rebekah K. Morano: Rebekah K. Morano completed her master's degree in music therapy at Western Michigan University. She received a Bachelor of Arts in Music and Psychology from Augustana College in Rock Island, IL in May 2018. During her time at Western Michigan, she worked as a research assistant in the Brain Interdisciplinary Research and Neurosciences (BRAIN) Lab, assisting with interdisciplinary research pursuits investigating the connections between music and neuroscience. Her internship experience was at Metro Music Therapy, Inc. in Denver, CO, working with children and adults with a range of diagnoses using Neurologic Music Therapy (NMT) interventions. Contact: rebekah.k.gohl@wmich.edu

Jennifer Fiore: Jennifer Fiore, PhD, MT-BC is an Associate Professor of Music Therapy at Western Michigan University. She holds a Bachelors (emphasis in Music Therapy), Masters in Music Education (emphasis in Music Therapy), and a doctorate of Philosophy in Music Education (emphasis in Music Therapy) from the University of Kansas. Jennifer has over 11 years of clinical experience within hospice working with both pediatric and adult hospice and palliative care patients, bereavement camps, oncology, pediatric medical, and older adults. She supervised music therapy practicum students clinical work, and established a university affiliated internship in hospice. Jennifer is a consistent presenter at the local, regional, and national level, and chairs the American Music Therapy Association's Standards of Clinical Practice Committee. Her research interests include hospice and palliative care, oncology, and stress and coping, and she has published within the Journal of Music Therapy, Arts in Psychotherapy, and OMEGA-Journal of Death and Dying. Contact: jennifer.fiore@wmich.edu

Alycia J. Sterenberg Mahon: Alycia Sterenberg Mahon, MM, MT-BC is the current Brain Interdisciplinary Research and Neurosciences (BRAIN) Lab manager at Western 
Michigan University. She completed her undergraduate work at Wartburg College in music therapy and psychology. Following her internship, she received a master's in music therapy at Western Michigan University. Currently, Alycia is pursuing a doctoral degree in Evaluation, Measurement, and Research at Western Michigan University in which she has completed courses in qualitative, quantitative, and mixed methods; evaluation; and measurement. Alycia has 14 peer-reviewed presentations at regional, national, and international conferences. Alycia's interests include pursuing continued knowledge of research methodologies and advancing the field of music therapy through research. Contact: a.j.sterenbergmahon@gmail.com

\section{Author Note}

The authors declare no conflicts of interest. The authors would like to acknowledge Dr. David Smith, Dr. Ying Zeng, Dr. Ann Chapleau, Dr. Joshua D. Naranjo, Meghan Feeman, and all of their families for their guidance and support.

Correspondence concerning this article should be addressed to Edward A. Roth, 1903 West Michigan Avenue Kalamazoo, Michigan 49008-5434; Email: mailto:edward.roth@wmich.edu

\section{Appendix}

The appendices are available at the following links: Appendix A: https://voices.no/ index.php/voices/article/view/3055/3298. Appendix B: https://voices.no/index.php/ voices/article/view/3055/3299. Appendix C: https://voices.no/index.php/voices/article/view/3055/3300

\section{Notes}

1. All three studies were approved by the Institutional Review Board at Western Michigan Univeristy.

\section{References}

American Music Therapy Association. (2011). AMTA Education and Advisory Board (ETAB) advisory on acronyms. https://www.musictherapy.org/members/ AMTA_Advisory_on_Acronyms/

American Music Therapy Association. (2013, November 23). AMTA professional competencies. http://www.musictherapy.org/about/competencies/

American Music Therapy Association. (2017). AMTA standards for education and clinical training. http://www.musictherapy.org/members/edctstan/

American Music Therapy Association. (2017). National roster internship guidelines.https://www.musictherapy.org/careers/national_roster_internship_guidelines/

American Music Therapy Association. (2018). 2018 AMTA member survey \& workforce analysis.https://www.musictherapy.org/assets/1/7/18WorkforceAnalysis.pdf

American Music Therapy Association. (2019). Organization directory search. https://netforum.avectra.com/eweb/ DynamicPage.aspx SSite $=$ AMTA2\&WebCode $=$ OrgSearch

Brookins, L. M. (1984). The music therapy clinical intern: Performance skills, academic knowledge, personal qualities, and interpersonal skills necessary for a student seeking clinical training. Journal of Music Therapy, 21(4), 193-201.https://doi.org/10.1093/jmt/ 21.4.193

Certification Board for Music Therapists (CBMT). (n.d.). About us. https://www.cbmt.org/ about/

Certification Board for Music Therapists (CBMT). (2015). Scope of music therapy practice. https://www.cbmt.org/wp-content/uploads/2019/10/CBMT-AMTA-Scope-of-MusicTherapy-Practice.pdf 
Clements-Cortes, A. (2019). A sequential mixed-methods study of pre-professionals' understanding of the undergraduate music therapy internship. Journal of Music Therapy, 56(3), 209-239. https://doi.org/10.1093/jmt/thz006

Davis, W. B., \& Gfeller, K. E. (2008). Music therapy: Historical perspective. In W. B. Davis, K. E. Gfeller, and M. H. Thaut (Eds.), An introduction to music therapy theory and practice (pp. 17-40). American Music Therapy Association.

Davis, W. B., Gfeller, K. E., and Kahler, E. P. (2018). A historical perspective of music therapy. In A. Knight, B. LaGasse, and A. Clair (Eds.), Music therapy: An introduction to the profession (pp. 33-52). American Music Therapy Association.

de l'Etoile, S. (2000). The history of the undergraduate curriculum in music therapy. Journal of Music Therapy, 37(1), 51-71. https://doi.org/10.1093/jmt/37.1.51

Field, A. (2013). IBM SPSS Statistics (4th ed.). SAGE Publications Ltd.

Hodgetts, S., Hollis, V., Triska, O., Dennis, S., Madill, H., \& Taylor, E. (2007). Occupational therapy students' and graduates' satisfaction with professional education and preparedness for practice. Canadian Journal of Occupational Therapy, 74(3), 148-160. https://doi.org/ 10.1177\%2F000841740707400303

Hsiao, F. (2014). Gatekeeping practices of music therapy academic programs and internships: A national survey. Journal of Music Therapy, 51(2), 186-206. https://doi.org/10.1093/jmt/ thu010

Jenkins, C. (2013). Functional musicianship of music therapy students: Entering internships as perceived by internship directors. Music Therapy Perspectives, 31(2), 175-180. https://doi.org/10.1093/mtp/31.2.175

Kim, S. A. (2011). Predictors of acculturative stress among international music therapy students in the United States. Music Therapy Perspectives, 29(2), 126-132. https://doi.org/ $10.1093 / \mathrm{mtp} / 29.2 .126$

Knight, A. J. (2008). Music therapy internship supervisors and preinternship students: A comparative analysis of questionnaires. Journal of Music Therapy, 45(1), 75-92. https://doi.org/10.1093/jmt/45.1.75

Madsen, C., \& Kaiser, K. (1999). Pre-internship fears of music therapists. Journal of Music Therapy, 36(1), 17-25. https://doi.org/10.1093/jmt/36.1.17

Ruhanen, L., Robinson, R., \& Breakey, N. (2013). A foreign assignment: Internships and international students. Journal of Hospitality and Tourism Management, 20, 1-4. https://doi.org/10.1016/j.jhtm.2013.05.005

Tanguay, C. L. (2008). Supervising music therapy interns: A survey of AMTA national roster internship directors. Journal of Music Therapy, 45(1), 52-74. https://doi.org/10.1093/jmt/ 45.1.52

Wheeler, B. L. (2002). Experiences and concerns of students during music therapy practica. Journal of Music Therapy, 39(4), 274-304. https://doi.org/10.1093/jmt/39.4.274

Young, L. (2009). Multicultural issues encountered in the supervision of music therapy internships in the United States and Canada. The Arts in Psychotherapy, 36(4), 191-201. https://doi.org/10.1016/j.aip.2009.01.004https://doi.org/10.1016/j.aip.2009.01.004 